\title{
Shape index in metric spaces
}

\author{
by \\ Francisco R. Ruiz del Portal and José M. Salazar (Madrid)
}

\begin{abstract}
We extend the shape index, introduced by Robbin and Salamon and Mrozek, to locally defined maps in metric spaces. We show that this index is additive. Thus our construction answers in the affirmative two questions posed by Mrozek in [12]. We also prove that the shape index cannot be arbitrarily complicated: the shapes of $q$-adic solenoids appear as shape indices in natural modifications of Smale's horseshoes but there is not any compact isolated invariant set for any locally defined map in a locally compact metric ANR whose shape index is the shape of a generalized solenoid. We also show that, for maps defined in locally compact metric ANRs, the shape index can always be computed in the Hilbert cube. Consequently, the shape index is the shape of the inverse limit of a sequence $\left\{P_{n}, g_{n}\right\}$ where $P_{n}=P$ is a fixed ANR and $g_{n}=g: P \rightarrow P$ is a fixed bonding map.
\end{abstract}

1. Introduction. The problem of constructing an analogue to the homotopical Conley index for discrete dynamical systems, posed in Conley's book [2], was solved by Robbin and Salamon [15] for diffeomorphisms of a compact manifold. The main problem with using Conley's ideas in the discrete case is the absence of homotopies along the orbits of the flow. Robbin and Salamon use shape theory to overcome this problem. However the setting is quite restrictive and the bijectivity and differentiability of the maps are strongly used. Independently, Mrozek [11] introduced an algebraic (cohomological) invariant for homeomorphisms of locally compact metric spaces, and Mrozek and Rybakowski [14] extended Mrozek's ideas to arbitrary maps defined in metric spaces. Later Mrozek [12] presented a general scheme for the construction of several Conley type indices that unifies the results in [15] and [11] for locally defined maps in locally compact metric spaces. This simplification of the hypotheses is important because the theory can be applied to the study of Poincaré maps associated to periodic phenomena. Then Mrozek obtained, in a unified way and in this general setting, the

2000 Mathematics Subject Classification: 37B30, 54H20, 54C56.

Key words and phrases: semidynamical systems, Conley index, shape theory.

The first author has been supported by DGES. 
cohomological and shape indices. The shape index is introduced as the inverse limit of a certain inverse sequence in the pointed shape category $\left(\mathrm{Sh}_{*}\right)$. Szymczak [17] introduced, in the language of category theory, an index such that all variants of the Conley index are functors on this categorical definition. More recently Franks and Richeson [4], using the notions of filtration pairs and shift equivalences, introduced a Conley index that is equivalent to Szymczak's. However, for the class of inverse sequences that appear when computing the shape index, shape and shift equivalences are so close that it seems difficult to give examples where the induced indices do not coincide. On the other hand, shape is a very developed theory with well known invariants that make the study and computation of Conley index more accessible.

In [12], Mrozek posed two problems:

a) Is the shape index additive?

b) Can this theory be extended to arbitrary metric spaces?

The main obstacle for solving the second problem is that the shape and inverse limit functors do not commute if the spaces involved are not compact.

In Sections 3 and 4 of this paper we solve both problems in the affirmative, using Rybakowski's conditions as in [14]. We use resolution theory to prove that in our case the shape and inverse limit functors commute, even though our spaces are not compact.

Section 5 is dedicated to studying which shape types can appear as shape indices of compact isolated invariant sets of a semidynamical system. While for continuous dynamical systems on manifolds the Conley index is the homotopy type of a polyhedron, for the discrete case the shapes of nonmovable spaces can appear. In fact, we obtain the shapes of $q$-adic solenoids as the shape indices of natural modifications of Smale's horseshoes. Nevertheless, shape indices cannot be arbitrarily complex. The shapes of generalized solenoids are never realized in locally compact ANRs. The main tool for this is that for computations one can always assume that the dynamical system is defined in the Hilbert cube. Then we can always assume the existence of prismatic index pairs. Consequently, the shape index is the shape of the inverse limit of a sequence $\left\{P_{n}, g_{n}\right\}$ where $P_{n}=P$ is a fixed ANR and $g_{n}=g: P \rightarrow P$ is a fixed bonding map.

In order to make this paper as selfcontained as possible, we begin by recalling briefly the basic notions of shape theory that we will use. The main reference is the book of Mardešić and Segal [9].

2. Basic notions of shape theory. Let $C$ be a category and let pro-C be the category whose objects are inverse systems in $C$ and whose morphisms are equivalence classes of morphisms of inverse systems with respect to the equivalence relation given in $[9, \mathrm{p} .7]$. 
Let $\mathrm{HTop}_{*}$ be the homotopy category of pointed topological spaces and $P=\mathrm{HPol}_{*}$ the full subcategory of $\mathrm{HTop}_{*}$ whose objects are the spaces which have the homotopy type of ANRs.

Definition 1 ([9, p. 19]). Let $T$ be a category and let $P$ be a subcategory of $T$. Given an object $X \in T$, a $T$-expansion of $X$ (with respect to $P$ ) is a morphism

$$
\mathbf{p}: X \rightarrow \mathbf{X}=\left(X_{\lambda}, p_{\lambda \lambda^{\prime}}, \Lambda\right)
$$

in pro- $T$ with the following universal property:

For any inverse system $\mathbf{Y}=\left(Y_{\beta}, q_{\beta \beta^{\prime}}, B\right)$ in the subcategory $P$, and any morphism $\mathbf{h}: X \rightarrow \mathbf{Y}$ in pro- $T$, there is a unique morphism $\mathbf{f}: \mathbf{X} \rightarrow \mathbf{Y}$ in pro- $T$ such that $\mathbf{h}=\mathbf{f} \circ \mathbf{p}$.

We say that $\mathbf{p}$ is a $P$-expansion of $X$ if $\mathbf{X}$ and $\mathbf{f}$ are in pro- $P$.

If $T=\mathrm{HTop}_{*}$ and $P=\mathrm{HPol}_{*}$, then every object $(X, *)$ in $\mathrm{HTop}_{*}$ admits an $\mathrm{HPol}_{*}$-expansion.

The objects of the pointed shape category, $\mathrm{Sh}_{*}$, are pointed topological spaces and given topological spaces $(X, *)$ and $(Y, *)$, a shape morphism is an equivalence class of morphisms in pro- $\mathrm{HPol}_{*}$ between $(\mathbf{X}, *)$ and $(\mathbf{Y}, *)$, with respect to a certain equivalence relation $([9$, p. 25]), where $\mathbf{p}:(X, *) \rightarrow$ $(\mathbf{X}, *)=\left(\left(X_{\lambda}, *\right), p_{\lambda \lambda^{\prime}}, \Lambda\right)$ and $\mathbf{q}:(Y, *) \rightarrow(\mathbf{Y}, *)=\left(\left(Y_{\beta}, *\right), q_{\beta \beta^{\prime}}, B\right)$ are fixed $\mathrm{HPol}_{*}$-expansions of $(X, *)$ and $(Y, *)$ respectively.

Two pointed spaces $(X, *)$ and $(Y, *)$ have the same (pointed) shape if they are isomorphic in $\mathrm{Sh}_{*}$; we will then write $S(X, *)=S(Y, *)$. The shape categories of unpointed topological spaces as well as that of pairs of topological spaces are introduced in a similar way.

Definition $2([9$, pp. 74,86$])$. Let $(X, *)$ be a pointed topological space. A resolution of $(X, *)$ is an inverse system $(\mathbf{X}, *)=\left(\left(X_{\lambda}, *\right), p_{\lambda \lambda^{\prime}}, \Lambda\right) \in$ pro-Top $_{*}$ and a morphism $\mathbf{p}:(X, *) \rightarrow(\mathbf{X}, *)$ in pro-Top $_{*}$ with the following two properties:

$(\mathrm{R} 1)$ Let $(P, *)$ be a pointed ANR, $\mathcal{V}$ an open covering of $P$ and $h$ : $(X, *) \rightarrow(P, *)$ a map. Then there is $\lambda \in \Lambda$ and a map $f:\left(X_{\lambda}, *\right) \rightarrow(P, *)$ such that $f \circ p_{\lambda}$ and $h$ are $\mathcal{V}$-near.

(R2) Let $(P, *)$ be a pointed ANR and $\mathcal{V}$ be an open covering of $P$. Then there is an open covering $\mathcal{V}^{\prime}$ of $P$ with the following property: If $\lambda \in \Lambda$ and $f, f^{\prime}:\left(X_{\lambda}, *\right) \rightarrow(P, *)$ are maps such that $f \circ p_{\lambda}$ and $f^{\prime} \circ p_{\lambda}$ are $\mathcal{V}^{\prime}$-near then there exists $\lambda^{\prime} \geq \lambda$ such that $f \circ p_{\lambda \lambda^{\prime}}$ and $f^{\prime} \circ p_{\lambda \lambda^{\prime}}$ are $\mathcal{V}$-near.

Denote by $H: \mathrm{Top}_{*} \rightarrow \mathrm{HTop}_{*}$ the homotopy functor. Resolutions are of interest because if $\mathbf{p}:(X, *) \rightarrow(\mathbf{X}, *)$ is a resolution of $(X, *)$ then $H(\mathbf{p}):(X, *) \rightarrow H((\mathbf{X}, *))$ is an $\mathrm{HTop}_{*}$-expansion of $(X, *)([9$, Theorem 2, p. 75]). 
Theorem $1([9$, p. 79$])$. Let $\mathbf{p}:(X, *) \rightarrow(\mathbf{X}, *)$ be a morphism in pro-Top $_{*}$. Assume that $\mathbf{p}$ has the following properties:

(B1) Let $\lambda \in \Lambda$ and let $U$ be an open subset of $X_{\lambda}$ containing $\operatorname{cl}\left(p_{\lambda}(X)\right)$. Then there is $\lambda^{\prime} \geq \lambda$ such that $p_{\lambda \lambda^{\prime}}\left(X_{\lambda^{\prime}}\right) \subseteq U$.

(B2) For every normal covering $\mathcal{U}$ of $X$ there is $\lambda \in \Lambda$ and a normal covering $\mathcal{V}$ of $X_{\lambda}$ such that $p_{\lambda}^{-1}(\mathcal{V})$ refines $\mathcal{U}$.

Then $\mathbf{p}$ is a resolution.

The continuity theorem is one of our basic tools.

Theorem $2([9$, p. 28$])$. Let $\mathbf{q}:(X, *) \rightarrow(\mathbf{X}, *)$ be an HTop $_{*}$-expansion of $(X, *) \in \mathrm{HTop}_{*}$. Then $\mathbf{q}$ is the inverse limit of $(\mathbf{X}, *)$ in the pointed shape category.

3. Shape index in metric spaces. Let $X$ be a metric space. Let $f: U \rightarrow X$ be a (continuous) map where $U$ is an open subset of $X$. A map $\sigma: J \rightarrow U \subset X$, where $J$ is an interval in $\mathbb{Z}$, is called a solution of $f$ if $f(\sigma(i-1))=\sigma(i)$ for every $i-1, i \in J$. If $0 \in J$ and $\sigma(0)=x$, we say that $\sigma$ is a solution through $x$.

Let $N \subset U$. The sets

$$
\begin{aligned}
\operatorname{Inv}^{+}(N) & =\left\{x \in X: f^{i}(x) \in N \text { for every } i \in \mathbb{N}\right\}, \\
\operatorname{Inv}^{-}(N) & =\left\{x \in X: \text { there exists a solution } \sigma: \mathbb{Z}_{-} \rightarrow N \text { through } x\right\}, \\
\operatorname{Inv}(N) & =\operatorname{Inv}^{+}(N) \cap \operatorname{Inv}^{-}(N)
\end{aligned}
$$

are called the positively invariant, negatively invariant and invariant sets with respect to $f$ in $N$, respectively.

Definition 3. A subset $A \subset U$ is called invariant with respect to $f$ if $\operatorname{Inv}(A)=A$. In a similar way, we say that $A$ is positively invariant if $\operatorname{Inv}^{+}(A)=A$ and negatively invariant if $\operatorname{Inv}^{-}(A)=A$.

Definition 4. A subset $N \subset U$ is admissible if for any pair of sequences $\left\{x_{n}: n \in \mathbb{N}\right\}$ and $\left\{m_{n}: n \in \mathbb{N}\right\}$ such that $\left\{f^{i}\left(x_{n}\right): 1 \leq i \leq m_{n}\right\} \subset N$ for every $n$ and $\left\{m_{n}\right\} \rightarrow \infty$, there exists an accumulation point of $\left\{f^{m_{n}}\left(x_{n}\right)\right.$ : $n \in \mathbb{N}\}$. In particular, every compact subset of $U$ is admissible.

Proposition 1. If $N \subset U$ is admissible then $\operatorname{Inv}^{-}(N), \operatorname{Inv}(N)$ are compact. If $\left\{x_{n}: n \in \mathbb{N}\right\}$ and $\left\{m_{n}: n \in \mathbb{N}\right\}$ are such that $\left\{f^{i}\left(x_{n}\right): 1 \leq i\right.$ $\left.\leq m_{n}\right\} \subset N$ for every $n$ and $\left\{m_{n}\right\} \rightarrow \infty$, then every accumulation point of $\left\{f^{m_{n}}\left(x_{n}\right): n \in \mathbb{N}\right\}$ belongs to $\operatorname{Inv}^{-}(N)$.

Definition 5. Let $K \subset U$ be an invariant set. Assume that $N$ is an admissible neighborhood of $K$ such that $K=\operatorname{Inv}(N)$. Then we say that $K$ is an isolated invariant set and $N$ is said to be an admissible isolating neighborhood of $K$. 
REMARK. If $K$ is an isolated invariant set then $K$ is compact. $\emptyset$ is an admissible isolating neighborhood of itself.

For the notion of index pair associated to an isolated invariant set we will adopt the definition of [14, Definitions 4.1 and 4.2].

Definition 6. Let $A \subset N \subset U$. We say that $A$ is $N$-positively invariant if $f(A) \cap N \subset A$.

Let $N$ be an admissible isolating neighborhood of a compact isolated invariant set $K$. The pair of closed subsets of $N, P=\left(P_{1}, P_{2}\right)$, is an index pair of $K$ in $N$ (with respect to $f$ ) if the following properties are satisfied:

a) $P_{1}, P_{2}$ are $N$-positively invariant.

b) $K \subset \operatorname{int}\left(P_{1} \backslash P_{2}\right)$.

c) $\operatorname{cl}\left(P_{1} \backslash P_{2}\right) \subset \operatorname{int}(N) \cap f^{-1}(\operatorname{int}(N))$.

$\operatorname{IP}(N)$ will denote the class of index pairs of $N$.

The next results are contained in [14] and their proofs are identical for locally defined maps.

Theorem 3 ([14, Theorem 4.4]). Let $N$ and $N^{\prime}$ be admissible isolating neighborhoods of $K$ such that $N \subset \operatorname{int}\left(N^{\prime}\right) \cap f^{-1}\left(\operatorname{int}\left(N^{\prime}\right)\right)$. Then, for every open neighborhood $W$ of $K$, there exists $P \in \operatorname{IP}(N)$ such that $\operatorname{cl}\left(P_{1} \backslash P_{2}\right) \subset W$.

COROLlary 1. For every compact isolated invariant set $K$ there exists a small enough admissible isolating neighborhood $N$ such that $\operatorname{IP}(N) \neq \emptyset$.

As in [12, p. 28], we consider the category of pairs Prs. In our case, the objects are pairs $P=\left(P_{1}, P_{2}\right)$ of topological spaces such that $P_{2} \subset P_{1}$ is a closed subset. We also have a covariant functor Quot $: \operatorname{Prs} \rightarrow$ Top $_{*}$.

Let $f: U \rightarrow X$ be a locally defined map. Let $P, Q \in$ Prs be closed subsets of $X$ such that $P_{1} \subset U$. Define $f_{P Q}: P_{1} \cap f^{-1}\left(Q_{1}\right) \ni x \mapsto f(x) \in Q_{1}$.

This map is not in general a morphism in Prs. Sufficient conditions for $f_{P Q}$ to be a morphism in Prs are given in the next proposition.

Proposition 2 ([12, p. 31]). Let $f$ be such that:

1) $P_{1} \backslash P_{2} \subset f^{-1}\left(Q_{1}\right)$.

2) $P_{2} \cap f^{-1}\left(Q_{1}\right) \subset f^{-1}\left(Q_{2}\right)$.

Then $f_{P Q} \in \operatorname{Prs}(P, Q)$.

Let $f: U \subset X \rightarrow X$ be a locally defined map and let $N \subset U$ be an admissible isolating neighborhood of a compact invariant set $K$. We can associate, to any $P=\left(P_{1}, P_{2}\right) \in \operatorname{IP}(N)$, a pair in Prs (that we will denote again by $P)$ defined as $P=\left(P_{1}, P_{1} \cap P_{2}\right) \in$ Prs. It is easy to see that $f_{P P} \in \operatorname{Prs}(P, P)$.

The homotopy relation of morphisms in Prs is introduced as in [12, p. 30]. If $f$ and $g$ are homotopic in $\operatorname{Prs}$ then $\operatorname{Quot}(f)$ and $\operatorname{Quot}(g)$ are homotopic 
in $\operatorname{Top}_{*}([12$, Prop. 5.7]). HPrs will denote the homotopy category of pairs defined in the obvious way.

$f_{P}=\operatorname{Quot}\left(f_{P P}\right):\left(P_{1} / P_{2},\left[P_{2}\right]\right) \rightarrow\left(P_{1} / P_{2},\left[P_{2}\right]\right)$ is a morphism in Top ${ }_{*}$. Define Quot $\left(\left[f_{P P}\right]\right)=\left[f_{P}\right]$, with $\left[f_{P P}\right]$ and $\left[f_{P}\right]$ the corresponding classes in HPrs and $\mathrm{HTop}_{*}$ respectively.

Let $S: \mathrm{HTop}_{*} \rightarrow \mathrm{Sh}_{*}$ be the shape functor (see [9, p. 26]) and let

$$
T=S \circ \text { Quot }: \operatorname{HPrs} \rightarrow \mathrm{Sh}_{*} \text {. }
$$

Now we are in a position to introduce the shape index. Let $X$ be a metric space. Let $f: U \subset X \rightarrow X$ be a locally defined map and let $N \subset U$ be an admissible isolating neighborhood of a compact invariant set $K$. Let $P$ be an index pair of $K$ in $N$.

Definition 7. We define the shape index of $K, C_{\lim , S}(K, f)$, as

$$
C_{\lim , S}(K, f)=\left(\lim S[(\mathbf{X}, *)], \lim \left(\left\{S\left(\left[f_{P}\right]\right)\right\}_{n}\right)\right)
$$

where $S[(\mathbf{X}, *)]=\left(\left(P_{1} / P_{2},\left[P_{2}\right]\right)_{n}, S\left(\left[f_{P}\right]\right), \mathbb{N}\right) \in \operatorname{pro}^{-S S}$ and $\left\{S\left(\left[f_{P}\right]\right)\right\}_{n}$ is a level morphism ([9, p. 12]) from $S[(\mathbf{X}, *)]$ to itself.

We will say that the pairs $(Y, g)$ and $\left(Y^{\prime}, g^{\prime}\right)$ (where $Y, Y^{\prime} \in$ Top $_{*}$ and $g: Y \rightarrow Y, g^{\prime}: Y^{\prime} \rightarrow Y^{\prime}$ are morphisms in $\left.\mathrm{Sh}_{*}\right)$ are isomorphic if there is a shape isomorphism $\varphi: Y \rightarrow Y^{\prime}$ such that $\varphi \circ g=g^{\prime} \circ \varphi$. In particular, if $(Y, g)$ and $\left(Y^{\prime}, g^{\prime}\right)$ are isomorphic, then $S(Y)=S\left(Y^{\prime}\right)$.

Our next purpose is to check that the above definition is consistent, i.e.:

1) $\lim S[(\mathbf{X}, *)]$ exists (and consequently $\lim \left(\left\{S\left(\left[f_{P}\right]\right)\right\}_{n}\right)$ exists).

2) $\left(\lim S[(\mathbf{X}, *)], \lim \left(\left\{S\left(\left[f_{P}\right]\right)\right\}_{n}\right)\right)$ does not depend, up to isomorphism, on the previous choices of $N$ and $P \in \operatorname{IP}(N)$.

As we said in the introduction, to check 1), Mrozek [12] used the commutativity of the shape and inverse limit functors (Continuity Theorem) for inverse systems of compact spaces. Now, if the local compactness of $X$ is not required, the spaces of the inverse systems in the last definition are not compact and in this case, in general, the shape and inverse limit functors do not commute. However, the admissibility of the isolating neighborhoods is enough to get (closed) index pairs $P=\left(P_{1}, P_{2}\right)$ for which the above commutativity holds.

Lemma 1. Let $P=\left(P_{1}, P_{2}\right) \in \operatorname{IP}(N)$ be an index pair associated to $K$. Then the inverse limit in $\operatorname{Top}_{*},(X, *)$, of the inverse system $\left(\left(P_{1} / P_{2},\left[P_{2}\right]\right)_{n}\right.$, $\left.f_{P}, \mathbb{N}\right)$ exists and it is compact.

Proof. Let

$$
Q=\left(Q_{1}, Q_{2}\right)=\left(\operatorname{Inv}^{-}(N) \cap P_{1}, \operatorname{Inv}^{-}(N) \cap P_{2}\right) .
$$

It is easy to see that $Q \in \operatorname{Prs}$ and, by Proposition $2, f_{Q Q} \in \operatorname{Prs}(Q, Q)$. Thus $\operatorname{Quot}\left(f_{Q Q}\right)=f_{Q}$ is continuous. 
Let $\left(X^{\prime}, *\right) \in \mathrm{Top}_{*}$ be the inverse limit of the system $\left(\left(Q_{1} / Q_{2},\left[Q_{2}\right]\right)_{n}\right.$, $\left.f_{Q}, \mathbb{N}\right)$. Then $X^{\prime}$ is compact because $Q_{1} / Q_{2} \neq \emptyset$ is compact.

Consider now the inclusion (in pro-Top ${ }_{*}$ )

$$
\mathbf{j}=\left(j_{n}, \phi\right):\left(\left(Q_{1} / Q_{2},\left[Q_{2}\right]\right)_{n}, f_{Q}, \mathbb{N}\right) \rightarrow\left(\left(P_{1} / P_{2},\left[P_{2}\right]\right)_{n}, f_{P}, \mathbb{N}\right)
$$

where $\phi=$ id. The following diagram commutes:

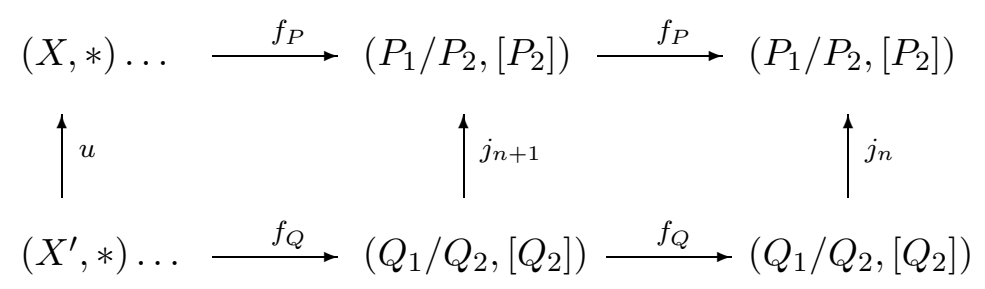

where $\left(X^{\prime}, *\right) \stackrel{u}{\longrightarrow}(X, *)$ is defined as $u\left(\left(\left[x_{n}^{\prime}\right]\right)_{n}\right)=\left(j_{n}\left(\left[x_{n}^{\prime}\right]\right)\right)_{n}$.

It is enough to check that $u$ is onto (in fact $u$ is a homeomorphism). Given $\left(\left[x_{n}\right]\right)_{n} \neq *$ with $\left(\left[x_{n}\right]\right)_{n} \in X \subset \prod_{n}\left(P_{1} / P_{2}\right)_{n}$ there is $n_{0} \geq 0$ such that $\left[x_{n}\right]=*$ for every $n \leq n_{0}$, and $\left[x_{n}\right] \neq *$ for every $n>n_{0}$.

Then $x_{n} \in P_{1} \backslash P_{2}$ for all $n>n_{0}$ with $f\left(x_{n}\right)=x_{n-1}$. Therefore $x_{n} \in$ $\operatorname{Inv}^{-}(N) \cap\left(P_{1} \backslash P_{2}\right)$ for every $n>n_{0}$.

Take $\left(\left[x_{n}^{\prime}\right]\right)_{n}$ where $\left[x_{n}^{\prime}\right]=\left[x_{n}\right]$ for every $n>n_{0}$ and $\left[x_{n}^{\prime}\right]=\left[Q_{2}\right]$ for $n \leq n_{0}$. It is clear that $u\left(\left(\left[x_{n}^{\prime}\right]\right)_{n}\right)=\left(\left[x_{n}\right]\right)_{n}$ with $\left(\left[x_{n}^{\prime}\right]\right)_{n} \in X^{\prime}$.

Proposition 3. Let $P$ be an index pair such that $P_{2} \subset P_{1}$. Consider the inverse system $(\mathbf{X}, *)=\left(\left(P_{1} / P_{2},\left[P_{2}\right]\right)_{n}, f_{P}, \mathbb{N}\right)$ and the inverse limit in $\mathrm{Top}_{*}$

$$
(X, *) \ldots \stackrel{f_{P}}{\longrightarrow}\left(P_{1} / P_{2},\left[P_{2}\right]\right) \stackrel{f_{P}}{\longrightarrow}\left(P_{1} / P_{2},\left[P_{2}\right]\right) .
$$

Then the corresponding morphism $\mathbf{p}:(X, *) \rightarrow(\mathbf{X}, *)$ in pro-Top $_{*}$ is a resolution.

Proof. It suffices to prove the properties (B1) and (B2) of Theorem 1. Let us see (B1).

If there exist $n \in \mathbb{N}$ and $U$ such that (B1) is not true, then for all $m \geq n$, $Y_{m}=\left(P_{1} / P_{2}\right) \backslash\left(f_{P}\right)_{n m}^{-1}(U) \neq \emptyset$.

Since $\left(f_{P}\right)_{m m^{\prime}}\left(Y_{m^{\prime}}\right) \subseteq Y_{m}$, we obtain $\mathbf{Y}=\left(Y_{m}, q_{m m^{\prime}}, M\right)$, where $q_{m m^{\prime}}=$ $\left.\left(f_{P}\right)_{m m^{\prime}}\right|_{Y_{m^{\prime}}}$ and $M=\{m \in \mathbb{N}: m \geq n\}$. We have $* \notin Y_{m}$ for all $m$. Thus we can identify $Y_{m}$ with a subset of $P_{1} \backslash P_{2}$ for all $m$.

The inclusion map $i_{m}: Y_{m} \rightarrow\left(P_{1} / P_{2}\right)_{m}, m \in M$, gives us a morphism $\mathbf{i}: \mathbf{Y} \rightarrow \mathbf{X}^{\prime}$ of inverse systems where $\mathbf{X}^{\prime}=\left(\left(P_{1} / P_{2}\right)_{m}, f_{P}, M\right)$. Let $X^{\prime}$ be the inverse limit of $\mathbf{X}^{\prime}$ (as in $\mathbf{X}$ ), and let $Y$ be the inverse limit of $\mathbf{Y}$. Let us see that $Y \neq \emptyset$ :

Since $Y_{m} \neq \emptyset$ for $m \geq n$, we can take $y_{n+1} \in Y_{n+1}, y_{n+2} \in Y_{n+2}, \ldots$ Therefore $\left(f_{P}\right)_{m m^{\prime}}\left(y_{m^{\prime}}\right)=\left(f_{P}\right)^{m^{\prime}-m}\left(y_{m^{\prime}}\right) \in Y_{m}$. 
We have $\left\{y_{n+1}, y_{n+2}, \ldots\right\}=\left\{\left[x_{1}\right],\left[x_{2}\right], \ldots\right\}$ for some $x_{m} \in\left(P_{1} \backslash P_{2}\right) \subset P_{1}$ admissible. The sequences $\{m: m \in \mathbb{N}\}$ and $\left\{x_{m}: m \in \mathbb{N}\right\}$ are such that $\left\{f^{i}\left(x_{m}\right): 1 \leq i \leq m\right\} \subset P_{1} \backslash P_{2} \subset P_{1}$ for all $m$, and $m \rightarrow \infty$. Since $P_{1}$ is admissible, the sequence $\left\{f^{m}\left(x_{m}\right)\right\}_{m}$ has a convergent subsequence and, by Proposition 1, there exists an accumulation point $x_{0} \in \operatorname{Inv}^{-}\left(P_{1}\right)$.

It is easy to see that $f_{P}^{m}\left(\left[x_{m}\right]\right)=\left[f^{m}\left(x_{m}\right)\right] \in Y_{n} \subset\left(P_{1} / P_{2}\right)_{n}$. Since $x_{0}$ is an accumulation point of $\left\{f^{m}\left(x_{m}\right)\right\}_{m},\left[x_{0}\right]$ is an accumulation point of $\left\{f_{P}^{m}\left(\left[x_{m}\right]\right)\right\}_{m}$. On the other hand, as $f_{P}^{m}\left(\left[x_{m}\right]\right) \in Y_{n}\left(\right.$ closed in $\left.\left(P_{1} / P_{2}\right)_{n}\right)$ for all $m$, it follows that $\left[x_{0}\right] \in Y_{n}$, and $x_{0} \in P_{1} \backslash P_{2}$.

We have $x_{0} \in \operatorname{Inv}^{-}\left(P_{1}\right) \backslash P_{2}$. Let $\left\{x_{m}^{\prime}\right\}$ be a sequence in $P_{1}$ such that $f\left(x_{k}^{\prime}\right)=x_{k-1}^{\prime}$ and $f\left(x_{1}^{\prime}\right)=x_{0}$. It is easy to see that $\left\{x_{m}^{\prime}\right\} \subset \operatorname{Inv}^{-}\left(P_{1}\right) \backslash P_{2}$ and therefore $f_{P}^{k}\left(\left[x_{k}^{\prime}\right]\right)=\left[f^{k}\left(x_{k}^{\prime}\right)\right]=\left[x_{0}\right] \in Y_{n}$ for all $\left[x_{k}^{\prime}\right] \in\left(P_{1} / P_{2}\right)_{n+k}$. Then $\left[x_{k}^{\prime}\right] \in Y_{n+k}$ for all $k$. We have $f_{P}\left(\left[x_{k}^{\prime}\right]\right)=\left[f\left(x_{k}^{\prime}\right)\right]=\left[x_{k-1}^{\prime}\right]$.

In this way we construct $y=\left(\left[x_{k}^{\prime}\right]\right)_{k} \in Y$, the inverse limit of $\mathbf{Y}$, and thus $Y \neq \emptyset$.

Let us consider the following inverse systems and morphisms:

$\mathbf{i}: \mathbf{Y} \rightarrow \mathbf{X}^{\prime}$, inclusion.

$\mathbf{j}: \mathbf{X} \rightarrow \mathbf{X}^{\prime}$ with $j_{m}=$ id $:\left(P_{1} / P_{2}\right)_{m} \rightarrow\left(P_{1} / P_{2}\right)_{m}$ for all $m \in M$.

$\mathbf{q}: Y \rightarrow \mathbf{Y}$, inverse limit of the inverse system $\mathbf{Y}$.

$\mathbf{p}: X \rightarrow \mathbf{X}$, inverse limit of the inverse system $\mathbf{X}$.

$\mathbf{p}: X^{\prime} \rightarrow \mathbf{X}^{\prime}$, inverse limit of the inverse system $\mathbf{X}^{\prime}$.

$i_{0}: Y \rightarrow X^{\prime}$, inverse limit of $\mathbf{i}, \lim (\mathbf{i})=i_{0}$.

$j_{0}: X \rightarrow X^{\prime}$, inverse limit of $\mathbf{j}, \lim (\mathbf{j})=j_{0}$.

Let $y \in Y$. Then $\left(p_{n} \circ i_{0}\right)(y)=\left(i_{n} \circ q_{n}\right)(y) \in i_{n}\left(Y_{n}\right)=\left(P_{1} / P_{2}\right) \backslash U$. On the other hand, $\left(p_{n} \circ i_{0}\right)(y)=\left(p_{n} \circ j_{0}\right)(x)=p_{n}(x) \in U$ for some $x \in X$, and this is a contradiction. The proof of (B1) is thus finished.

Let us see (B2). For every $x \in X$, we can select $m(x) \in \mathbb{N}$ and an open set $W_{x} \subseteq\left(P_{1} / P_{2}\right)_{m(x)}$ such that $x \in\left(p_{m(x)}\right)^{-1}\left(W_{x}\right) \subseteq U_{x}$ for some $U_{x} \in \mathcal{U}$ (see [9, Remark 3, p. 58]). By Lemma $1, X$ is compact and there exists a finite set $\left\{m_{1}, \ldots, m_{n}\right\} \subset \mathbb{N}$ and open subsets $W_{i} \subseteq\left(P_{1} / P_{2}\right)_{m_{i}}$ such that $\left\{\left(p_{m_{i}}\right)^{-1}\left(W_{i}\right)\right\}_{i \in\{1, \ldots, n\}}$ is an open covering of $X$ which refines $\mathcal{U}$. Take $m \geq m_{1}, \ldots, m_{n}$ and set $V_{i}=\left(\left(f_{P}\right)_{m_{i} m}\right)^{-1}\left(W_{i}\right), i \in\{1, \ldots, n\}$. Then $\mathcal{V}=\left\{V_{1}, \ldots, V_{n},\left(P_{1} / P_{2}\right)_{m} \backslash p_{m}(X)\right\}$ is an open covering of $\left(P_{1} / P_{2}\right)_{m}$ and $\left(p_{m}\right)^{-1}(\mathcal{V})=\left\{\left(p_{m}\right)^{-1}\left(V_{1}\right), \ldots,\left(p_{m}\right)^{-1}\left(V_{n}\right)\right\}$ refines $\mathcal{U}$.

Since paracompactness is preserved for closed maps (see Michael's theorem in [3]) and since the projection $q_{P}: P_{1} \rightarrow P_{1} / P_{2}$ is closed we find that $P_{1} / P_{2}$ is paracompact and hence $\mathcal{V}$ is normal.

Corollary 2. The inverse limit $\mathbf{p}:(X, *) \rightarrow(\mathbf{X}, *)$, which is a resolution of $(X, *)$, is such that $H(p):(X, *) \rightarrow H((\mathbf{X}, *))$ is an $\mathrm{HTop}_{*^{-}}$ 
expansion. Consequently, $S[(\mathbf{X}, *)]$ admits an inverse limit, $\lim S[(\mathbf{X}, *)]=$ $S(\lim (\mathbf{X}, *))=S((X, *))$.

The proof follows automatically from Theorem 2 .

To guarantee the consistence of $C_{\lim , S}(K, f)$ we only have to check that condition 2) is satisfied, i.e. $\left(\lim S[(\mathbf{X}, *)], \lim \left(\left\{S\left(\left[f_{P}\right]\right)\right\}_{n}\right)\right)$ does not depend, up to isomorphism, on the previous choices of $N$ and $P \in \operatorname{IP}(N)$. This proof, which we do not give here, needs various steps which appear, slightly modified, in [14, Section 5].

Remark. The shape index of the empty set is $C_{\lim , S}(\emptyset, f)=(*, \mathrm{id})$. It suffices to get the index pair $P=\left(P_{1}, P_{2}\right)=(\emptyset, \emptyset)$. If the shape index in the admissible isolating neighborhood $N$ is not $(*$,id), we have $K \neq \emptyset$.

\section{Main properties of the shape index. Additivity}

Definition 8 . Let $\left(Y_{1}, *\right),\left(Y_{2}, *\right) \in$ Top $_{*}$ be pointed topological spaces. We denote by $\left(Y_{1}, *\right) \vee\left(Y_{2}, *\right) \in \operatorname{Top}_{*}$ the union of $Y_{1}$ and $Y_{2}$ with the base points identified. If we have pointed maps (morphisms of $\operatorname{Top}_{*}$ ) $h_{1}$ : $\left(Y_{1}, *\right) \rightarrow\left(Y_{1}, *\right)$ and $h_{2}:\left(Y_{2}, *\right) \rightarrow\left(Y_{2}, *\right)$, we define the map $h_{1} \vee h_{2}:$ $\left(Y_{1}, *\right) \vee\left(Y_{2}, *\right) \rightarrow\left(Y_{1}, *\right) \vee\left(Y_{2}, *\right)$ by setting $\left(h_{1} \vee h_{2}\right)\left(y_{i}\right)=h_{i}\left(y_{i}\right)$ for $y_{i} \in Y_{i}, i \in\{1,2\}$. The map $h_{1} \vee h_{2}$ is a morphism of $\mathrm{Top}_{*}$.

THEOREM 4. Let $K$ be an isolated invariant set which is the disjoint sum of two isolated invariant sets $K_{1}$ and $K_{2}$. Then $C_{\lim , S}(K, f)$ is the coproduct $C_{\lim , S}\left(K_{1}, f\right) \vee C_{\lim , S}\left(K_{2}, f\right)$. More precisely,

$$
C_{\lim , S}(K, f)=\left(S(\lim (\mathbf{X}, *) \vee \lim (\mathbf{Y}, *)), S\left(\left[\lim \left(\left\{f_{P}\right\}_{n}\right) \vee \lim \left(\left\{f_{Q}\right\}_{n}\right)\right]\right)\right)
$$

where

$$
(\mathbf{X}, *)=\left(\left(P_{1} / P_{2}, *\right)_{n}, f_{P}, \mathbb{N}\right), \quad(\mathbf{Y}, *)=\left(\left(Q_{1} / Q_{2}, *\right)_{n}, f_{Q}, \mathbb{N}\right)
$$

with $P=\left(P_{1}, P_{2}\right) \in \operatorname{IP}\left(N_{1}\right)$ and $Q=\left(Q_{1}, Q_{2}\right) \in \operatorname{IP}\left(N_{2}\right)$, for $N_{1}$ and $N_{2}$ small enough isolating neighborhoods of $K_{1}$ and $K_{2} ;\left\{f_{P}\right\}_{n}$ and $\left\{f_{Q}\right\}_{n}$ are level morphisms of $(\mathbf{X}, *)$ and $(\mathbf{Y}, *)$ in themselves.

Proof. Let $U_{1}, U_{2}$ be open sets in $U$ such that $K_{1} \subset U_{1}, K_{2} \subset U_{2}$ and $\operatorname{cl}\left(U_{1}\right) \cap \operatorname{cl}\left(U_{2}\right)=\emptyset$.

For $i=1,2$ we select admissible isolating neighborhoods $N_{i}$ of $K_{i}$ such that $N_{i} \subset U_{i} \cap f^{-1}\left(U_{i}\right)$. It is not difficult to see that $N_{1} \cup N_{2}$ is an admissible isolating neighborhood of $K_{1} \cup K_{2}$.

Let $P \in \operatorname{IP}\left(N_{1}\right)$ and $Q \in \operatorname{IP}\left(N_{2}\right)$. It is an exercise to prove that $P \cup Q=$ $\left(P_{1} \cup Q_{1}, P_{2} \cup Q_{2}\right)$ is an index pair of $K$ in $N_{1} \cup N_{2}$.

Observe that

$$
\left(\left(P_{1} \cup Q_{1}\right) /\left(P_{2} \cup Q_{2}\right), *\right) \simeq\left(P_{1} / P_{2}, *\right) \vee\left(Q_{1} / Q_{2}, *\right) .
$$


We define the pointed map

$$
f_{P} \vee f_{Q}:\left(P_{1} / P_{2}, *\right) \vee\left(Q_{1} / Q_{2}, *\right) \rightarrow\left(P_{1} / P_{2}, *\right) \vee\left(Q_{1} / Q_{2}, *\right) .
$$

It is clear, by the construction of $N_{1}$ and $N_{2}$, that

$$
f_{P \cup Q} \equiv f_{P} \vee f_{Q}
$$

determines a topological conjugation.

Let

$$
\begin{aligned}
(\mathbf{Z}, *) & =\left(\left(\left(P_{1} \cup Q_{1}\right) /\left(P_{2} \cup Q_{2}\right), *\right)_{n}, f_{P \cup Q}, \mathbb{N}\right), \\
(\mathbf{W}, *) & =\left(\left(\left(P_{1} / P_{2}, *\right) \vee\left(Q_{1} / Q_{2}, *\right)\right)_{n}, f_{P} \vee f_{Q}, \mathbb{N}\right) .
\end{aligned}
$$

It is easy to see that $\lim (\mathbf{W}, *)=\lim (\mathbf{X}, *) \vee \lim (\mathbf{Y}, *)$. Then

$$
\begin{aligned}
C_{\lim , S}(K, f) & =\left(\lim S[(\mathbf{Z}, *)], \lim \left(\left\{S\left(\left[f_{P \cup Q}\right]\right)\right\}_{n}\right)\right) \\
& =\left(S(\lim (\mathbf{Z}, *)), S\left(\left[\lim \left(\left\{f_{P \cup Q}\right\}_{n}\right)\right]\right)\right) \\
& =\left(S(\lim (\mathbf{W}, *)), S\left(\left[\lim \left(\left\{f_{P} \vee f_{Q}\right\}_{n}\right)\right]\right)\right) \\
& =\left(S(\lim (\mathbf{X}, *) \vee \lim (\mathbf{Y}, *)), S\left(\left[\lim \left(\left\{f_{P}\right\}_{n}\right) \vee \lim \left(\left\{f_{Q}\right\}_{n}\right)\right]\right)\right) .
\end{aligned}
$$

The second equality (up to isomorphism) follows from Corollary 2. The equality of morphisms

$$
\lim \left(\left\{S\left(\left[f_{P \cup Q}\right]\right)\right\}_{n}\right)=S\left(\left[\lim \left(\left\{f_{P \cup Q}\right\}_{n}\right)\right]\right)
$$

is obvious by the uniqueness of the inverse limit morphism.

The homotopy property of the shape index follows from the results of $[14$, Section 6$]$, and we will not give its proof here.

Let $f: \Lambda \times U \rightarrow X$ be a continuous map with $\Lambda \subset \mathbb{R}$ a compact interval. We denote by $f_{\lambda}: U \rightarrow X$ the partial map $f_{\lambda}(x)=f(\lambda, x)$.

If $J$ is a subinterval of $\Lambda$ then we consider the map

$$
f_{J}: J \times U \ni(\lambda, x) \mapsto\left(\lambda, f_{\lambda}(x)\right) \in J \times X .
$$

We denote by $\operatorname{Inv}\left(N, f_{\lambda}\right)$ the invariant set with respect to $f_{\lambda}$ in $N$.

Theorem 5 (Homotopy property, [14, p. 167]). Let $f: \Lambda \times U \rightarrow X$ be a continuous map and let $N \subset U$ be a closed subset of $X$ such that for all $\mu \in \Lambda$ there exists a neighborhood $J$ of $\mu$ in $\Lambda$ with $J \times N \subset J \times U$ being an admissible isolating neighborhood with respect to $f_{J}$ in $J \times X$. Then $C_{\lim , S}\left(\operatorname{Inv}\left(N, f_{\lambda}\right), f_{\lambda}\right)$ is independent of $\lambda \in \Lambda$.

Let $X, Y$ be metric spaces with open subsets $U \subset X$ and $V \subset Y$. Let $\varphi: U \rightarrow Y$ and $\psi: V \rightarrow X$ be continuous maps such that $K \subset U$ is an isolated invariant set with respect to $f=\psi \circ \varphi$.

Theorem 6 (Commutativity property). $\varphi(K)$ is an isolated invariant set with respect to $g=\varphi \circ \psi$. Moreover, $C_{\lim , S}(K, f)=C_{\lim , S}(\varphi(K), g)$.

Proof. For the first assertion, it is enough to take $M, N$ of the proof of [12, p. 35], with $M \subset \operatorname{dom}(f)=\varphi^{-1}(V)$ being an admissible isolating 
neighborhood of $K$ with respect to $f$. The equality $\operatorname{Inv}(N, g)=\varphi(K)$ is proved in [12]. The proof of the admissibility of $N$ is simple.

For the second assertion, let $\psi^{\prime}: \psi^{-1}(U) \rightarrow U$ be the restriction of $\psi$. We define

$$
f_{0}=\psi^{\prime} \circ \varphi: \varphi^{-1}\left(\psi^{-1}(U)\right) \rightarrow U, \quad g_{0}=\varphi \circ \psi^{\prime}: \psi^{-1}(U) \rightarrow Y .
$$

We have $g_{0}=g$ and $C_{\lim , S}(K, f)=C_{\lim , S}\left(K, f_{0}\right)$.

Let $N$ be an admissible isolating neighborhood of $\varphi(K)$ for $g$. It is not hard to see that $\varphi^{-1}(N)$ is an admissible isolating neighborhood of $K$ for $f_{0}$.

Let $\left(Q_{1}, Q_{2}\right) \in \operatorname{IP}(N)$ with $Q_{2} \subset Q_{1}$. We define $P_{1}=\varphi^{-1}\left(Q_{1}\right)$ and $P_{2}=\varphi^{-1}\left(Q_{2}\right)$. It is not difficult to prove that $P=\left(P_{1}, P_{2}\right) \in \operatorname{IP}\left(\varphi^{-1}(N)\right)$.

For the rest of the proof see [12].

5. Shape types which can appear as shape indices. While the shape (homotopic) index for continuous dynamical systems on manifolds is the shape (homotopic) type of a compact polyhedron $([15])$, the index for the discrete case can be more complicated.

For all $n \in \mathbb{N}$ take $S_{n}^{1}=\{z \in \mathbb{C}:|z|=1\}$ with base point $1=z_{n} \in S_{n}^{1}$. Fot $q \in \mathbb{Z}$ the (pointed) $q$-adic solenoid, $\left(S_{q}, *\right)$, is the inverse limit of the inverse system $\left(\left(S_{n}^{1}, z_{n}\right), q_{n}, \mathbb{N}\right)$ where the pointed maps $q_{n}:\left(S_{n+1}^{1}, 1\right) \rightarrow$ $\left(S_{n}^{1}, 1\right)$ are defined as $q_{n}(z)=z^{q}$ for all $n \in \mathbb{N}$.

If $\left\{d_{n}\right\}_{n \in \mathbb{N}}$ is a sequence of integers and we take pointed maps $h_{n}$ : $\left(S_{n+1}^{1}, 1\right) \rightarrow\left(S_{n}^{1}, 1\right), h_{n}(z)=z^{d_{n}}$ for all $n \in \mathbb{N}$, then the inverse limit is a generalized solenoid.

In this section $(T, *)$ denotes a pointed generalized solenoid obtained from a sequence $\left\{d_{n}\right\}_{n \in \mathbb{N}}$ of mutually prime integers. The solenoids are compact, connected, non-movable (pointed) spaces.

Our aim is to obtain the shape of the $q$-adic solenoids as the shape index of isolated invariant sets of discrete dynamical systems (modifying Smale's G-horseshoe). On the other hand, the shape of a generalized solenoid $T$ is not the index of an isolated invariant set of a locally defined map in a locally compact metric ANR. Thus, the index is not arbitrarily complex. We prove that, in this context, the shape index is the shape of the inverse limit of an inverse system $\left(\left(P_{n}, z_{n}\right), g_{n}, \mathbb{N}\right)$ where, for all $n \in \mathbb{N},\left(P_{n}, z_{n}\right)=(P, *)$ and $g_{n}=g$, with $P$ a fixed finite polyhedron.

If $f: N \subset X \rightarrow X$ is a continuous map and $K \subset \operatorname{int}(N)$ is an isolated invariant set, we denote by $S(K, f)$ the shape type (without morphism) of $C_{\lim , S}(K, f)$.

Proposition 4. For all $q \in \mathbb{N}$ there exists a locally defined diffeomorphism $f_{q}:[0,1]^{2} \rightarrow \mathbb{R}^{2}$ and an isolated invariant set $K_{q}$ for $f_{q}$ such that $S\left(K_{q}, f_{q}\right)=S\left(S_{q}, *\right)$. The case $q=2$ is obtained from Smale's G-horseshoe. 
Proof. Let us prove that $S\left(K_{2}, f_{2}\right)=S\left(S_{2}, *\right)$. Let $\left(G_{2}, *\right)$ be a (pointed) compact set obtained as the inverse limit of the inverse sequence associated to an index pair $P=\left(P_{1}, P_{2}\right)$ such that $P_{1} / P_{2}$ is homotopically equivalent to the pointed union of two circles (see Figure 1 ).

We have to prove that $S\left(G_{2}, *\right)=S\left(S_{2}, *\right)$. The solenoid $\left(S_{2}, *\right)$ is the inverse limit of the inverse sequence

$$
\ldots \stackrel{z^{2}}{\longrightarrow}\left(S^{1}, 1\right) \stackrel{z^{2}}{\longrightarrow}\left(S^{1}, 1\right) \stackrel{z^{2}}{\longrightarrow}\left(S^{1}, 1\right) .
$$

On the other hand, $\left(G_{2}, *\right)$ is the inverse limit of the inverse sequence

$$
\ldots \stackrel{\phi}{\longrightarrow}\left(S^{1} \vee S^{1}, *\right) \stackrel{\phi}{\longrightarrow}\left(S^{1} \vee S^{1}, *\right) \stackrel{\phi}{\longrightarrow}\left(S^{1} \vee S^{1}, *\right)
$$

where $\phi:\left(S^{1} \vee S^{1}, *\right) \rightarrow\left(S^{1} \vee S^{1}, *\right)$ is a pointed map which transforms the paths $a$ and $b$ into $a b$ (see Figure 1).

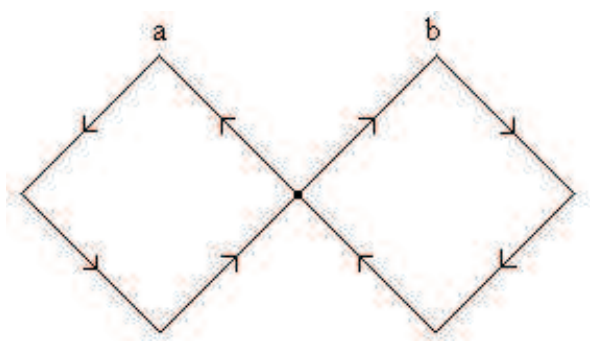

Fig. 1

We define

$$
\psi=\left(\operatorname{id}_{S^{1}}, \operatorname{id}_{S^{1}}\right):\left(S^{1} \vee S^{1}, *\right) \rightarrow\left(S^{1}, 1\right) .
$$

Let $\varrho:\left(S^{1}, 1\right) \rightarrow\left(S^{1} \vee S^{1}, *\right)$ be a pointed map, morphism of HTop $*$, which transforms the path identity $S^{1} \rightarrow S^{1}$ into the path $a b$.

We therefore have the following commutative diagram (up to pointed homotopy):

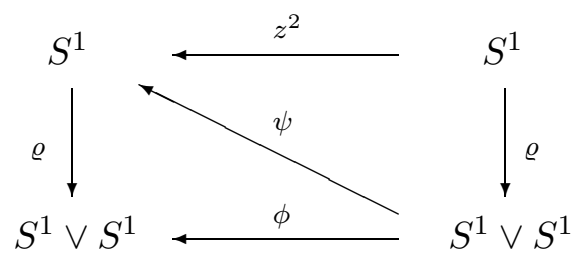

Now, using Morita's Theorem for level isomorphisms in pro-categories $([9$, p. 112]), we finish the proof for the case of $q=2$. 
Let $q$ be arbitrary. Take the locally defined diffeomorphism $f_{q}:[0,1]^{2} \rightarrow$ $\mathbb{R}^{2}$ as in Figure 2 .

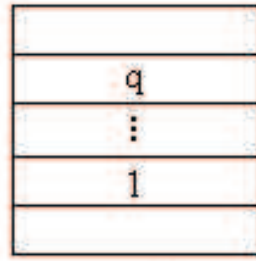

$\mathrm{I}^{2}$
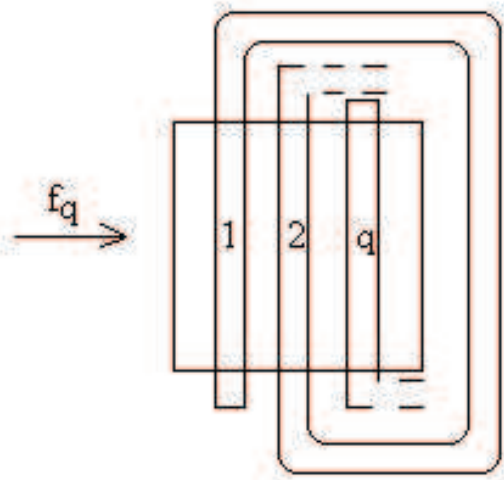

Fig. 2

The proof is analogous to the case of $q=2$.

We use, in our context, the techniques of [18, Lemma 5.1] (see also [16]) to guarantee the existence of suitable index pairs for isolated invariant sets with respect to semidynamical systems defined in $Q=\prod_{n=1}^{\infty}[0,1 / n]$, the Hilbert cube.

Let $f: Q \rightarrow Q$ be a continuous map. For $\varepsilon>0$ set $\mathcal{A}(\varepsilon)=\left\{A \cap Q: A=\prod_{i=1}^{\infty} A_{i}, \forall i \exists k \in \mathbb{N}\right.$ with $A_{i}=k \varepsilon$ or $\left.A_{i}=[k \varepsilon,(k+1) \varepsilon]\right\}$.

We define the multivalued maps $T_{\varepsilon}, F_{\varepsilon}: Q \rightarrow \mathcal{P}(Q)$ as

$$
T_{\varepsilon}(x)=\bigcup\{A \in \mathcal{A}(\varepsilon): x \in A\}, \quad F_{\varepsilon}(x)=\left(T_{\varepsilon} \circ f \circ T_{\varepsilon}\right)(x) .
$$

We say that a set $A \subset Q$ is a prism if there exist $m \in \mathbb{N}$ and a finite polyhedron $P$ such that $A=P \times Q \subset \prod_{n=1}^{m}[0,1 / n] \times Q$ (see [1, p. 104]).

Lemma 2 ([16, Lemma 2]). Let $F: I \times Q \times \mathbb{Z} \rightarrow \mathcal{P}(Q)$ be the multivalued map

$$
F(\varepsilon, x, n)=F_{\varepsilon}^{n}(x) .
$$

Then $F$ is a compact valued upper semicontinuous map such that for all $\varepsilon \in I, F_{\varepsilon}: Q \times \mathbb{Z} \rightarrow \mathcal{P}(Q)$ is a discrete multivalued dynamical system in the sense of Kaczynski and Mrozek [8].

Proposition 5. Let $f: Q \rightarrow Q$ be a continuous map and let $K$ be an isolated invariant set. Then there exists an admissible isolating neighborhood $M$ of $K$ and an index pair $\left(Q_{1}, Q_{2}\right) \in \operatorname{IP}(M)$ such that $Q_{1}$ and $Q_{2}$ are prisms. 
Proof. The proof is based on that of [18, Lemma 5.1], using the last lemma and [8, Theorem 2.5 and Lemmas 2.6 and 2.7]. We modify the construction of [18] in that we select a prismatic index pair for $K$. We need an admissible isolating neighborhood $M$ of $K$ and a pair $\left(Q_{1}, Q_{2}\right) \in \operatorname{IP}(M)$ of compact sets (prisms) in $M$.

We define $M=N_{\varepsilon}$ as in [18]. Consider $\left(P_{1}, P_{2}\right)$ as in [18] such that

$$
\operatorname{cl}\left(P_{1} \backslash P_{2}\right) \subset \operatorname{int}\left(N_{\varepsilon}\right) \cap f^{-1}\left(\operatorname{int}\left(N_{\varepsilon}\right)\right)=W .
$$

Let $d \in \mathbb{N}$. We define $\left(Q_{1}, Q_{2}\right)$ as

$$
Q_{1}=T_{\varepsilon / d}\left(P_{1}\right) \cap N_{\varepsilon}, \quad Q_{2}=T_{\varepsilon / d}\left(P_{2}\right) \cap N_{\varepsilon} .
$$

If $d$ is large enough it is not difficult to prove that $\left(Q_{1}, Q_{2}\right) \in \operatorname{IP}(M)$.

TheOREM 7. Let $f: U \subset X \rightarrow X$ be a continuous map with $X$ a locally compact metric $A N R$ and $U$ an open subset of $X$. Then for every isolated invariant set $K, S(K, f)=S(\lim (\mathbf{X}, *))$ for $(\mathbf{X}, *)=\left(\left(Q_{1} / Q_{2}, *\right)_{n}, g_{Q}, \mathbb{N}\right)$, where $\left(Q_{1}, Q_{2}\right)$ is a prismatic index pair of $K$ with respect to a continuous map $g: Z \subset Q \rightarrow Q$ extending $f$.

Proof. Consider $X$ to be embedded as a closed subset in a normed space $B$. Let $N$ be a compact isolating neighborhood of $K$. Take a retraction $r: U^{X} \subset B \rightarrow X$ where $U^{X}$ is an open neighborhood of $X$ in $B$. From [5], there is a compact ANR $A^{N}$ such that $N \subset A^{N} \subset U^{X}$.

Let $V$ be an open subset of $X$ such that $K \subset V \subset N$ and $f(V) \subset N$. Define $W=r^{-1}(V) \cap A^{N}$ and consider the map

$$
f_{1}=f \circ r_{\mid W}: W \subset A^{N} \rightarrow A^{N} .
$$

Using the commutativity property we see that $S(K, f)=S\left(K, f_{1}\right)$.

Now we can assume that $A^{N} \subset Q$. Take a retraction

$$
r_{1}: U^{A^{N}} \subset Q \rightarrow A^{N}
$$

where $U^{A^{N}}$ is an open neighborhood of $A^{N}$ in $Q$. Let $Z=r_{1}^{-1}(W) \subset U^{A^{N}}$ and define

$$
g=f_{1} \circ r_{1 \mid Z}: Z \subset Q \rightarrow Q .
$$

Using commutativity again, we conclude that $S\left(K, f_{1}\right)=S(K, g)$.

Corollary 3. Let $f: U \subset X \rightarrow X$ be a continuous map with $X$ a locally compact metric ANR. Then $S(K, f) \neq S(T, *)$ for every isolated invariant set $K$.

Proof. We have $S(K, f)=S\left(\lim \left(\left(Q_{1} / Q_{2},\left[Q_{2}\right]\right)_{n}, g_{Q}, \mathbb{N}\right)\right)=S(Z, *)$, with $(Z, *)$ the inverse limit.

If $S(K, f)=S(T, *)$, then $S(T, *)=S(Z, *)$. There exist $l \in \mathbb{N}$ and pointed maps $\alpha:\left(Q_{1} / Q_{2},\left[Q_{2}\right]\right) \rightarrow\left(S^{1}, 1\right), \beta:\left(S^{1}, 1\right) \rightarrow\left(Q_{1} / Q_{2},\left[Q_{2}\right]\right)$ such that 


$$
\beta \circ \alpha \simeq g_{Q}^{l}:\left(Q_{1} / Q_{2},\left[Q_{2}\right]\right) \rightarrow\left(Q_{1} / Q_{2},\left[Q_{2}\right]\right)
$$

where $\simeq$ stands for the pointed homotopy relation.

Therefore, we have the following commutative diagram (up to pointed homotopy):

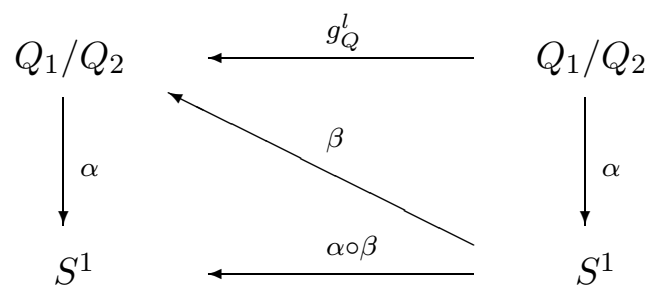

Thus $S(K, f)=S(Z, *)=S(T, *)=S\left(S_{q}, *\right)$ where $\left(S_{q}, *\right)$ is the pointed solenoid obtained as the inverse limit of

$$
\ldots\left(S^{1}, 1\right) \stackrel{\alpha \circ \beta}{\longrightarrow}\left(S^{1}, 1\right) \stackrel{\alpha \circ \beta}{\longrightarrow}\left(S^{1}, 1\right) .
$$

But this is a contradiction by [10, Theorem 17].

COROLlary 4. The shape index of an isolated invariant set of a locally defined map in a locally compact metric ANR is the shape of the inverse limit of an inverse sequence $\left(\left(P_{n}, z_{n}\right), g_{n}, \mathbb{N}\right)$ where, for all $n \in \mathbb{N},\left(P_{n}, z_{n}\right)=(P, *)$ is fixed and $g_{n}=g:(P, *) \rightarrow(P, *)$ is fixed with $P$ a finite polyhedron.

The polyhedron $P$ is finite because we can obtain $Q_{1} / Q_{2}$, a compact ANR (see [6, Theorem 1.2, p. 178]) that, by West's theorem [19], has the homotopy type of a finite polyhedron. Thus the index is the shape of a compact space of finite shape dimension.

Final remarks. From the last corollary we conclude that the shape of a space of infinite shape dimension, like the pointed union of spheres $\bigvee_{j=1}^{\infty} S^{j}$, is not the index of a compact invariant isolated set of a dynamical system in a locally compact metric ANR. Moreover, we can modify the proof of Corollary 3 to prove that the shape of simpler spaces, as the hawaiian earring $H$, of dimension 1 , is not the index in a dynamical system.

On the other hand, if the inverse sequence of polyhedra is pointed movable and the shape groups are countable, then the index is the shape of a FANR ([9, Theorem 18, p. 235]). It seems hard to find indices which are the shape of movable spaces and are not FANRs. In this sense, the example of Corollary 3 is interesting because it is a one-dimensional, non-movable continuum with trivial shape groups.

The last corollary is a generalized result about the finiteness of the Conley index type (see [18]). 


\section{References}

[1] K. Borsuk, Theory of Retracts, Monograf. Mat. 44, PWN, Warszawa, 1967.

[2] C. C. Conley, Isolated Invariant Sets and the Morse Index, CBMS Regional Conf. Ser. in Math. 38, Amer. Math. Soc. Providence, RI, 1978.

[3] R. Engelking, General Topology, Monograf. Mat. 60, PWN, Warszawa, 1977.

[4] J. Franks and D. Richeson, Shift equivalence and the Conley index, Trans. Amer. Math. Soc. 352 (2000), 3305-3322.

[5] J. Girolo, Approximating compact sets in normed linear spaces, Pacific J. Math. 98 (1992), 81-89.

[6] S. T. Hu, Theory of Retracts, Wayne State Univ. Press, Detroit, 1965.

[7] T. W. Hungerford, Algebra, Springer, New York, 1989.

[8] T. Kaczynski and M. Mrozek, Stable index pairs for discrete dynamical systems, Canad. Math. Bull. 40 (1997), 448-455.

[9] S. Mardešić and J. Segal, Shape Theory, North-Holland, Amsterdam, 1982.

[10] —, - Shapes of compacta and ANR-systems, Fund. Math. 72 (1971), 41-59.

[11] M. Mrozek, Leray functor and cohomological Conley index for discrete dynamical systems, Trans. Amer. Math. Soc. 318 (1990), 149-178.

[12] - Shape index and other indices of Conley type for local maps on locally compact Hausdorff spaces, Fund. Math. 145 (1994), 15-37.

[13] - Index pairs and the fixed point index for semidynamical systems with discrete time, ibid. 133 (1989), 179-194.

[14] M. Mrozek and K. P. Rybakowski, A cohomological Conley index for maps on metric spaces, J. Differential Equations 90 (1991), 143-171.

[15] J. W. Robbin and D. Salamon, Dynamical systems, shape theory and the Conley index, Ergodic Theory Dynam. Systems 8 (1988), 375-393.

[16] F. R. Ruiz del Portal and J. M. Salazar, Fixed point index in hyperspaces: A Conleytype index for discrete semidynamical systems, J. London Math. Soc. 64 (2001), 191-204.

[17] A. Szymczak, The Conley index for discrete semidynamical systems, Topology Appl. 66 (1995), 215-240.

[18] —, The Conley index and symbolic dynamics, Topology 35 (1996), 287-299.

[19] J. E. West, Mapping Hilbert cube manifolds to ANR's: a solution of a conjecture of Borsuk, Ann. of Math. 106 (1977), 1-18.

Francisco R. Ruiz del Portal

Departamento de Geometría y Topología

Facultad de Matemáticas

Universidad Complutense de Madrid

Madrid 28040, Spain

E-mail: R_Portal@mat.ucm.es
José Manuel Salazar Departamento de Matemáticas Universidad de Alcalá Alcalá de Henares Madrid 28871, Spain E-mail: josem.salazar@uah.es 